\title{
Bempedoic Acid: The New Kid on the Block for the Treatment of Dyslipidemia and LDL Cholesterol: A Narrative Review
}

\author{
Uazman Alam • Dalal Y. Al-Bazz $\cdot$ Handrean Soran
}

Received: January 27, 2021 / Accepted: April 30, 2021 / Published online: May 26, 2021

(C) The Author(s) 2021

\begin{abstract}
Diabetes is a major risk factor for atherosclerotic cardiovascular disease (ASCVD) in which dyslipidaemia plays a crucial role. Statins are first line therapy for primary and secondary prevention of ASCVD; however, adverse events include reversible musculoskeletal and liver side effects in addition to a diabetogenic association. In this short review, we provide a succinct narrative of the future role and current trial data of a novel first-in-class molecule, bempedoic acid. The authors provide their expert insight with a focus on Phase III randomised controlled trials
\end{abstract}

U. Alam $(\bowtie) \cdot$ D. Y. Al-Bazz

Department of Cardiovascular and Metabolic

Medicine and the Pain Research Institute, Clinical

Sciences Centre, Institute of Life Course and

Medical Sciences, University of Liverpool, Liverpool

University Hospital NHS Foundation Trust,

Liverpool L9 7AL, UK

e-mail: Uazman.alam@liverpool.ac.uk;

Uazman.alam@manchester.ac.uk

U. Alam

Division of Diabetes, Endocrinology and

Gastroenterology, Institute of Human

Development, University of Manchester,

Manchester, UK

H. Soran $(\varangle)$

Institute of Cardiovascular Sciences, University of

Manchester and Manchester Foundation Trust,

Manchester M13 9WL, UK

e-mail: Handrean.Soran@mft.nhs.uk;

hsoran@aol.co.uk
(RCT) of bempedoic acid. Bempedoic acid was approved by the US Food and Drug Administration (FDA) and the European Medicines Agency (EMA) in February and March 2020, respectively, and is a novel molecule which inhibits cholesterol biosynthesis in the same mechanistic pathway as statins. It is a first-inclass small molecule, delivered as a prodrug and administered as an oral, once-daily dose that decreases low-density lipoprotein cholesterol (LDL-C) levels. Phase II and III RCTs have demonstrated efficacy with adequate safety data as mono- or combination therapy with statins and ezetimibe. Bempedoic acid is hepatically converted to the active drug with a lack of activation in skeletal muscle. Due to this novel mechanism, musculoskeletal-related adverse events exhibit a lower prevalence providing an alternative pharmacotherapy in statin-intolerant patients. Bempedoic acid may be used as an adjunct to diet and maximally tolerated statin therapy or in statin-intolerant patients for the treatment of dyslipidaemia. The recent National Institute of Health and Care Excellence (NICE) (UK) technology appraisal guidance [TA694] published in April 2021 recommended bempedoic acid with ezetimibe as a treatment option for primary hypercholesterolaemia or mixed dyslipidaemia if statins are not tolerated or contraindicated and if there is inadequate control of LDL-C with ezetimibe alone. Additionally, outcomes trials evaluating 'hard' endpoints 
in statin-intolerant patients or those with ASCVD are currently underway.

Keywords: Atherosclerotic cardiovascular disease; Bempedoic acid; Diabetes; Dyslipidaemia; Statins

\section{Key Summary Points}

Diabetes is a major risk factor for atherosclerotic cardiovascular disease (ASCVD) and dyslipidaemia is a major cause in establishing and/or worsening ASCVD.

Statins are the mainstay drug therapy for dyslipidaemia including in patients with diabetes.

Many patients on statin therapy do not receive the desired reduction in lowdensity lipoprotein cholesterol (LDL-C) particularly because of intolerance.

Bempedoic acid is a new lipid-lowering drug which offers an alternative to statin therapy.

The Cholesterol Lowering via Bempedoic Acid, an ACL-Inhibiting Regimen (CLEAR) trials have demonstrated efficacy in LDL-C reduction with preliminary data demonstrating no increase in new or worsening of established diabetes.

\section{DIGITAL FEATURES}

This article is published with digital features, including a summary slide, to facilitate understanding of the article. To view digital features for this article go to https://doi.org/10.6084/ m9.figshare.14510643. 
there has been a disparity about the true incidence of side effects of statins and possible harm from these side effects [16]. Randomised controlled clinical trials report very low rates of even the most well-authenticated side effects, such as myopathy [17]. However, in uncontrolled observational studies muscle symptoms are reported in as many as $10-20 \%$ of statin recipients [18-24]. Current evidence also suggests that statins are associated with a 'diabetogenic' potential by increasing the risk of newonset diabetes and worsening established diabetes through a modest increase in glycaemic levels [25-27].

Previous reports outlined the risk of newonset diabetes in statin treatment which was increased by $9 \%$ compared to those taking placebo, whilst intensive and cumulative statin therapy was also associated with an increased risk of diabetes development [26]. Although the mechanism by which statins increase the incidence of diabetes are not fully established, evidence suggests a multi-factorial cause involving the inhibition of $\beta$-cell glucose transporters, delayed ATP production, pro-inflammatory and oxidative $\beta$-cell effects of plasma-derived cholesterol, inhibition of calcium channel-dependent insulin secretion and $\beta$-cell apoptosis [25].

The paradox is raised wherein statins, which represent the strongest form of cardiovascular risk reduction, may promote progression to diabetes. However, the benefits of continued statin therapy very much outweigh the potential diabetogenic risks, the introduction of novel lipid-lowering agents may provide a possible solution for those at high risk of diabetes, e.g. prediabetes/metabolic syndrome groups with statin intolerance given its lack of deleterious effect on glycaemic control. Hence, there is a current clinical need for an effective alternative to statin therapy for LDL-C-lowering.

This short review provides a succinct narrative of the future role and current trial data of the novel first-in-class molecule, bempedoic acid, in the management of ASCVD and dyslipidaemia, with consideration of the excess of ASCVD in diabetes mellitus.

\section{METHODS}

The authors provide their expert insight; in addition, a literature search was undertaken on bempedoic acid in PubMed, Google Scholar and clinicaltrials.gov to ensure relevant trials were discussed. The authors primarily focussed on phase III randomised controlled trials (RCT) of bempedoic acid. All seven available phase III trials were reviewed, and four trials were chosen based on completion with published and analysed results. This article is based on previously conducted studies and does not contain novel data of animal and human study origin.

\section{RESULTS}

Of seven phase III trials that evaluated bempedoic acid, four have been completed and published: Cholesterol Lowering via Bempedoic Acid, an ACL-Inhibiting Regimen (CLEAR) Harmony (NCT02666664), CLEAR Wisdom (NCT02991118), CLEAR Tranquillity (NCT03001076) and CLEAR Serenity (NCT02988115). Table 1 outlines the primary characteristics of these studies. Of the remaining three trials, one is currently in process and two have been completed but are yet to be published and for the purpose of this review have not been discussed [28]. All of the available trial data demonstrate significant LDL-C reductions.

Phase III trials have demonstrated the efficacy of bempedoic acid $180 \mathrm{mg}$ once daily in reducing LDL-C compared to placebo. The CLEAR Harmony trial evaluated the efficacy and safety of bempedoic acid $180 \mathrm{mg}$ once daily $(n=1488)$ versus placebo $(n=742)$ in patients with ASCVD and/or heterozygous familial hypercholesterolaemia $(\mathrm{HeFH})$ receiving maximal dose of tolerated statins over 52 weeks [29]. At week 12, bempedoic acid reduced the mean LDL-C level by $19.2 \mathrm{mg} / \mathrm{dl}(0.50 \mathrm{mmol} / \mathrm{l})$ representing $16.5 \%$ reduction from baseline $(P<0.001)$. Adverse event and serious adverse event rates were similar in the bempedoic acid group compared to the placebo group (78.5 vs. $78.7 \%$ and $14.5 \%$ vs. $14.0 \%$ respectively). However, the incidence of adverse events leading to discontinuation was significantly higher 


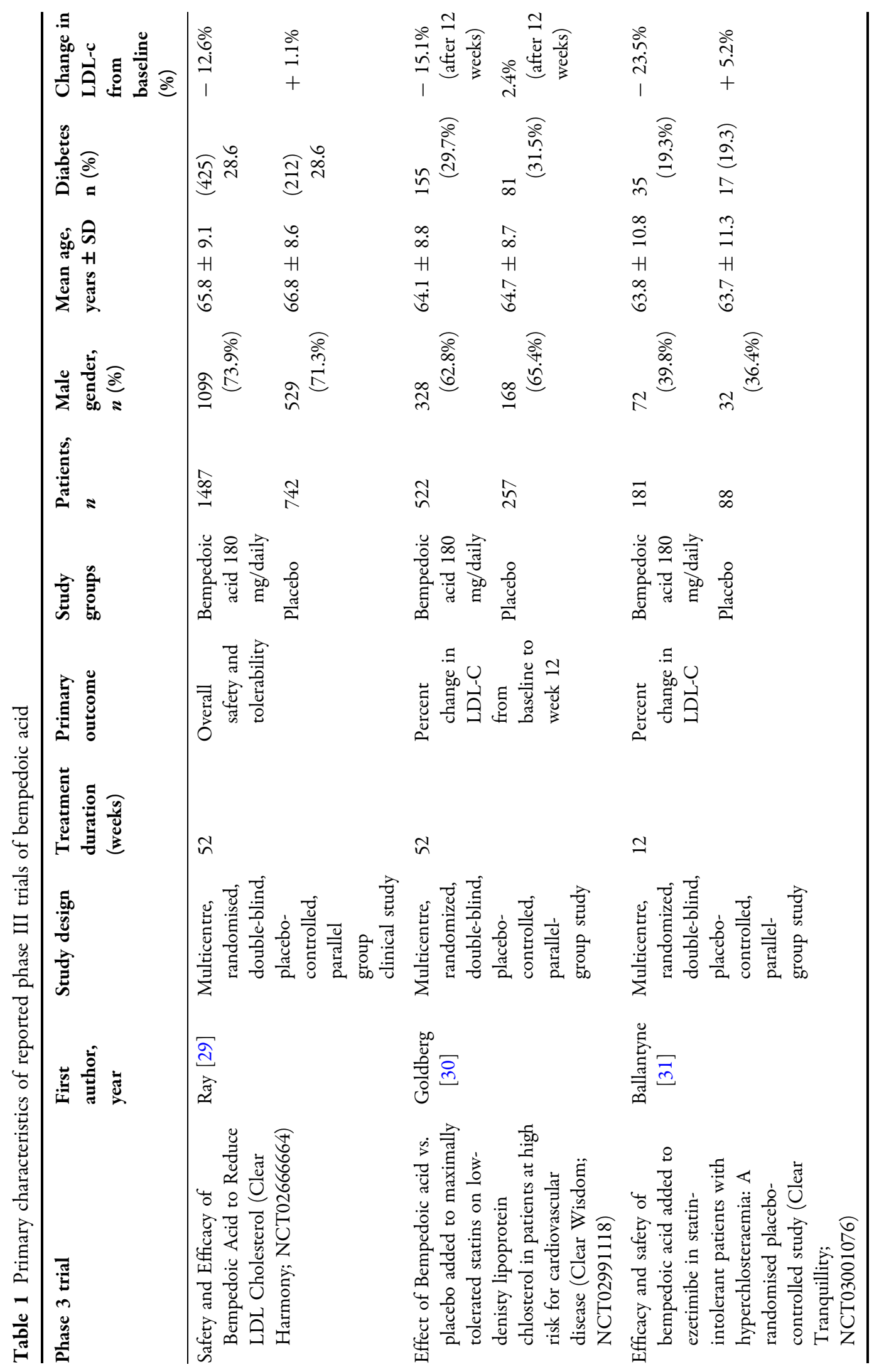




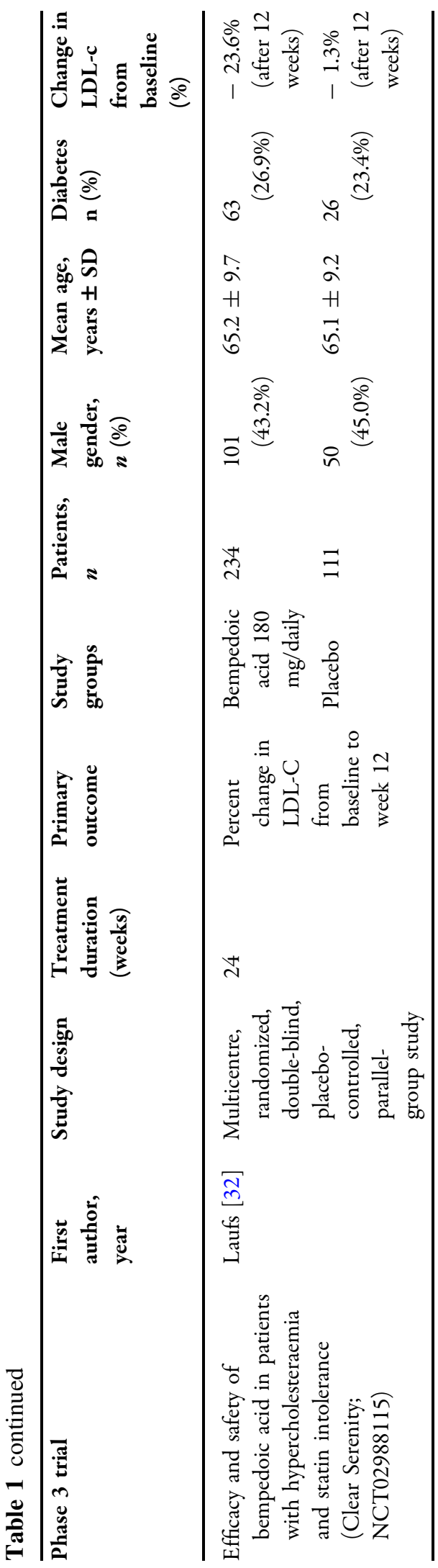

in the bempedoic acid group (10.9\%) than in the placebo group (7.1\%), as was the incidence of gout $(1.2 \%$ vs. $0.3 \%$ respectively). Similar data with a significant reduction of LDL-C with bempedoic acid $180 \mathrm{mg}$ once daily compared to placebo were reported in the CLEAR Wisdom trial.

CLEAR Harmony and CLEAR Wisdom assessed the safety and tolerability of $180 \mathrm{mg}$ once daily bempedoic acid versus placebo in patients with high cardiovascular risk and elevated LDL cholesterol not adequately controlled by their current maximally tolerated statin therapy $[29,30]$. Both trials were of 52 weeks duration. At 12 weeks bempedoic acid reduced the mean placebo corrected LDL-C by $-16.5 \%$ (CLEAR Harmony) and $-17.4 \%$ (CLEAR Wisdom) from baseline $[29,30]$ and showed no greater risk of adverse events compared to placebo CLEAR (Harmony; $78.5 \%$ versus $78.7 \%$, CLEAR Wisdom; $70.1 \%$ versus $70.8 \%$ ) $[29,30]$. CLEAR Tranquillity observed the safety and efficacy of bempedoic acid added to ezetimibe in statinintolerant patients with hypercholesterolaemia [31]. In this 12-week trial, bempedoic acid added to ezetimibe reduced LDL-C by $28.5 \%$ more than placebo $(p<0.001 ;-23.5 \%$ bempedoic acid, $+5.0 \%$ placebo) [31]. The efficacy and safety of bempedoic acid in patients with statin intolerance were also studied in the CLEAR Serenity trial of 24 weeks duration [32]. Patients with a history of intolerance two statins (one at the lowest available dose) were randomised (2:1) to either $180 \mathrm{mg}$ once daily bempedoic acid or placebo once daily [32]. At week 12 bempedoic acid treatment significantly lowered LDL-C by a placebo corrected $-21.4 \%(95 \% \mathrm{CI}-25.1$ to $-17.7 \%$; $p<0.001)$ [32].

Across all four trials bempedoic acid was well tolerated [33]. Common adverse events included gout, urinary tract infections, nasopharyngitis and hyperuricaemia [29-32]. In CLEAR Harmony, the incidence of new-onset diabetes or worsening diabetes was lower in the treatment arm compared to placebo $(n=49$ [3.3\%] vs. $\mathrm{n}=40$ [5.4\%]) [29]. CLEAR wisdom found that amongst patients with diabetes, HbA1c decreased by $0.08 \%$ from baseline in the bempedoic acid cohort, whilst levels increased 
by $0.13 \%$ in the placebo cohort [30]. Favourable glycaemic control included a reduction in worsening was consistent over the 1-year treatment period.

In CLEAR Tranquillity, $2.3 \%$ of patients in the placebo arm developed diabetes compared to $1.1 \%$ of patients in the intervention arm [31]. Similarly, new-onset or worsening diabetes was less commonly seen in the bempedoic acid treatment arm (2.1\%) compared to placebo (4.5\%) in the CLEAR Serenity trial [32]. Fasting glucose $\geq 126 \mathrm{mg} / \mathrm{dl}$ and glycosylated haemoglobin $\geq 6.5 \%(48 \mathrm{mmol} / \mathrm{mol})$ was less frequently observed in individuals in the bempedoic acid cohort regardless of pre-existing diabetes compared to placebo [32].

A recent meta-analysis combined the outcome of new-onset or worsening diabetes in these four trials which reported $3.7 \%$ of individuals taking bempodic acid compared to 5.7\% of individuals on placebo [34]. Bempodeic acid was associated with a risk reduction of 0.65 (95\% CI 0.44-0.96; $I^{2}=23 \%$ ) [34].

\section{DISCUSSION}

Bempedoic acid (NEXLETOL $^{\circledR}$ in the USA, Nilemdo ${ }^{\circledR}$ in the EU) (Diiachi Sankyo; Esperion) (ETC-1002; ESP55016) is a first-in-class small molecule, delivered as a prodrug and administered as an oral, once-daily dose that decreases LDL-C level. It was approved by both the US Food and Drug Administration (FDA) and the European Medicines Agency (EMA) in February and March 2020 respectively [35]. Bempedoic acid is administered orally in one $180 \mathrm{mg}$ dose licensed by the FDA as an addition to maximally tolerated statin treatment in adults with HeFH or established ASCVD who require additional lowering of LDL-C (US label). In the EU, the licensed indication differs slightly and is indicated in adults with primary hypercholesterolaemia (HeFH/non-familial) or mixed dyslipidaemia in combination with a statin with or without other lipid-lowering therapies in people unable to meet LDL-C targets with maximal statin treatment alone or in combination with other lipid-lowering therapies in people who are statin intolerant or for whom a statin is contraindicated (please see the British National Formulary: https://bnf.nice.org.uk/drug/ bempedoic-acid.html).

Bempedoic acid is a prodrug. The activated substance inhibits adenosine triphosphate citrate lyase, which is involved in the cholesterol biosynthesis in the liver upstream of $\beta$ hydroxy $\beta$-methylglutaryl-CoA (HMG-CoA) reductase, the enzyme that is antagonised by statins. Similar to statins, bempedoic acid depletes intracellular cholesterol leading to upregulation of hepatic LDL receptor expression, thus increasing the clearance of circulating LDL-C. However, this is where the similarities diverge and the activation through primarily liver enzymes (very long-chain acylCoA synthetase-1, which lacks expression in skeletal muscle) is postulated to lessen the risk of myopathy and musculoskeletal-related side effects. Experimental and clinical data suggest that bempedoic acid has anti-atherosclerotic effects [36], improvement in glucose homeaostasis [37], inflammation and blood pressure [38] in addition to its LDL-C-lowering activity [37]. Recently, a systematic review and meta-analysis of seven clinical trials confirmed its anti-inflammatory effect demonstrated by a reduction in high-sensitivity c-reactive protein (hsCRP) [mean difference compared to controls: $-13.2 \%$; $95 \%$ CI $-16.7 \%$ to $-9.79 \%$; $P<0.001]$. Given the accepted role of inflammation and hsCRP as a surrogate biomarker of systemic inflammation and the prediction of ASCVD [39], these findings may be of prognostic benefit, which needs to be further interrogated in in vivo mechanistic and post-marketing studies. Bempedoic acid's primary antiatherosclerotic benefit is through lowering LDLC; however, clearly its anti-inflammatory effect may be also clinically relevant. Interestingly, LDL-C reduction with evolocumab (a PCSK9 inhibitor) reduced absolute risk of ASCVD in patients with higher baseline hsCRP [40]. Also, in healthy persons without hyperlipidaemia but with high hsCRP levels, LDL-C lowering with rosuvastatin significantly reduced the incidence of major ASCVD events [41].

Lipid-lowering therapy targeting the reduction of LDL-C reduces the risk of cardiovascular disease and LDL-C also remains one of the best 
biomarkers for ASCVD. However, it should be noted that the value of LDL-C as a surrogate endpoint for ASCVD is not absolute given its heterogeneous nature with differences in outcomes in LDL-C-lowering drug effects [42]. For instance, the use of fibrates as lipid-lowering therapyhas demostrated no significant cardiovascular benefit [43]. Nonetheless, other drugs such as bempedoic acid and ezetimibe have shown favourable cardioprotective outcomes $[34,43]$. Given the undisputed causal relationship between LDL-C and ASCVD [8] with no known level below which further LDL-C lowering ceases to be beneficial [44], the significant LDL-C reduction with bempedoic acid provides clear hope and the expectation that these data will be translated into a reduction in ASCVD events and subsequent mortality. Importantly, the CLEAR Outcomes trial (NCT02993406) is precisely evaluating hard ASCVD endpoints in statin-intolerant patients or patients at high risk of ASCVD and is currently underway; its future results are eagerly awaited.

A number of phase III trials have shown the efficacy of bempedoic acid $180 \mathrm{mg}$ once daily in reducing LDL-C compared to placebo. This article has focussed on four phase III trials under the CLEAR trials umbrella including Harmony, Wisdom, Tranquillity and Serenity. Collectively evidence from these trials has shown reductions in LDL-C baseline of up to approximately $24 \%$ after 12 weeks and was generally well tolerated throughout the treatment length. Importantly, the magnitude of LDL-C reduction in the CLEAR Serenity trial is similar to that of lowintensity statins (simvastatin $10 \mathrm{mg}$, lovastatin $20 \mathrm{mg}$, pravastatin $20 \mathrm{mg}$, fluvastatin $40 \mathrm{mg}$ ) [6]. All of the aforementioned trials have demonstrated an improvement in systemic inflammation with a reduction in hsCRP and a reduction in new onset or worsening of diabetes. In addition to the CLEAR Tranquillity trial, another study has shown efficacy and safety in combination with ezetimibe [45]. Based on these data a fixed dose combination of bempedoic acid (180 $\mathrm{mg}$ ) and ezetimibe (10 $\mathrm{mg})$ (NEXLIZET $^{\circledR}$ in the US, Nustendi ${ }^{\circledR}$ in the EU) has been released [35]. Despite no direct headto-head comparison with statins, the overall musculoskeletal-related adverse events seem to be lower providing an alternative pharmacotherapy in statin-intolerant patients [46].

A further recent meta-analysis demonstrated a decreased risk of new-onset or worsening of type 2 diabetes (odds ratio 0.59; 95\% CI $0.39-0.90 ; P=0.01$ ) [33]. Clearly, this in contrast to statin therapy, which is associated with an small increase in the absolute risk of newonset type 2 diabetes [47]. Although the currently published CLEAR series of trials had reported improvement of hyperglycaemia and reduction of worsening or new-onset diabetes, most of the trials did not display a statistically significant trend. Also, across these trials, patients and events of interest were few. Indeed, people with diabetes ranged between 20 and $30 \%$ of the full trial cohorts, however there was no specific focus on prediabetic groups or groups at high risk of progession to diabetes. Further research in cohorts in metabolic syndrome or prediabetes with the specific intent to delineate any putative beneficial anti-glycaemic effect requires further study. Whilst bempedoic acid has demonstrated promising outcomes, it is important to note that statin therapy remains the primary lipid-lowering therapy according to international consensus [48-50].

Adherence to statins is a known challenge, particularly amongst patients taking low-intensity treatment. A large retrospective cohort study reported adherence levels of $84 \%$ after 1 year of treatment in patients on high-intensity treatment with CVD compared with adherence of only $57 \%$ in patients on low-intensity treatment [51]. After 6 years of therapy, adherence rates in both groups dropped further to $72 \%$ and $48 \%$ in the high- and low-intensity cohorts respectively [51]. These results were supported by a more recent study which retrospectively observed patients from 2009 to 2016; overall, approximately $53 \%$ of patients were adherent to statin therapy [52]. Adherence and discontinuation were related to intensity levels with patients on high-intensity therapy being more adherent (63.7\%) and less likely to discontinue treatment compared to those on moderate- or low-intensity therapy [52].

The adherence to therapy is fundamental to maximal possible reduction of cardiovascular risk. Average risk reduction of cardiovascular 
events is about $40 \%$ and $22 \%$ in patients adhering to high- or low-intensity statin therapy respectively [51]. This is compared to a risk reduction of only $10 \%$ in high-intensity treatment and 5\% in low-intensity treatment in patients with poor compliance [51].

Currently injectable therapy, e.g. PCSK9 inhibitors such as evolocumab, is an alternative to statin therapy in patients with poor compliance. It is given every 2 weeks and indicated in patients with high cardiovascular risk and/or primary $\mathrm{HeFH}$ and persistently raised LDL-C [53]. However, its use is limited by cost [43]. The issue of cardiovascular risk benefit in relation to compliance poses the question of whether adherence and thus cardiovascular risk would be improved with newer and potentially, more cost-effective therapy options such as bempedoic acid. However, a meta-analysis observed a $37 \%$ discontinuation rate of bempedoic acid compared to placebo. There was no pattern that could explain the increased risk of discontinuation and thus this needs to be further investigated [33].

\section{CONCLUSIONS}

Currently, many patients do not achieve the desired reduction in LDL-C, particularly in severe hyperlipidaemias, in individuals who do not tolerate the first-line statin therapy and even in general diabetes cohorts [54]. Therefore, addition of bempedoic acid to the array of lipidlowering therapies is most welcome particularly given the high prevalence of ASCVD in diabetes mellitus. Indeed NICE technology appraisal guidance [TA694] has recommended bempedoic acid with ezetimibe as a treatment option for primary hypercholesterolaemia or mixed dyslipidaemia if statins are not tolerated or contraindicated and if there is inadequate control of LDL-C with ezetimibe alone. The approval of the novel first-in-class drug, bempedoic acid, has added to the armoury of lower LDL-C-lowering therapy available to physicians treating dyslipidaemia and ASCVD.

\section{ACKNOWLEDGEMENTS}

Funding. No funding or sponsorship was received for this study or publication of this article.

Authorship. All named authors meet the International Committee of Medical Journal Editors (ICMJE) criteria for authorship of this article, take responsibility for the integrity of the work as a whole, and have given their approval for this version to be published.

Authors' Contributions. All authors contributed to the study conception and design. Material preparation, data collection and analysis were performed by Uazman Alam, Dalal Yousif Al-Bazz and Handrean Soran. The first draft was completed by Uazman Alam and Handrean Soran and following peer review, editing and writing was completed by Dalal Yousif Al-Bazz. All authors commented on previous versions of the manuscript. All authors read and approved the final manuscript.

Disclosures. Uazman Alam has received support from Sanofi, Astra Zeneca, Eli Lilly and Diiachi Sankyo for the provision of educational meetings and is also a member of the journal's Editorial Board. Uazman Alam reports no other relevant disclosure. Dalal Yousif Al-Bazz has nothing to disclose. Handrean Soran received research grants and charitable donations from Alexion, Amryt, AKCEA, Amgen, Pfizer, MSD, Genzyme-Sanofi and MSD and personal fees and education grants from Aegerion, Amgen, Janssen Cilag Ltd, MSD, AKCEA, Pfizer, Sanofi, NAPP, Link-Medical, Alexion, Medicine's Company, Novartis and Diiachi Sankyo.

Compliance with Ethics Guidelines. This article is based on previously conducted studies and does not contain any new studies with human participants or animals performed by any of the authors.

Data Availability. Data sharing is not applicable to this article as no datasets were generated or analyzed during the current study. 
Open Access. This article is licensed under a Creative Commons Attribution-NonCommercial 4.0 International License, which permits any non-commercial use, sharing, adaptation, distribution and reproduction in any medium or format, as long as you give appropriate credit to the original author(s) and the source, provide a link to the Creative Commons licence, and indicate if changes were made. The images or other third party material in this article are included in the article's Creative Commons licence, unless indicated otherwise in a credit line to the material. If material is not included in the article's Creative Commons licence and your intended use is not permitted by statutory regulation or exceeds the permitted use, you will need to obtain permission directly from the copyright holder. To view a copy of this licence, visit http://creativecommons.org/licenses/by$\mathrm{nc} / 4.0 /$.

\section{REFERENCES}

1. Lee ET, Keen H, Bennett PH, Fuller JH, Lu M. Follow-up of the WHO multinational study of vascular disease in diabetes: general description and morbidity. Diabetologia. 2001;44(Suppl 2):S3-13. https://doi.org/10.1007/pl00002936. PMID: 11587047.

2. Howard BV, et al. Coronary heart disease risk equivalence in diabetes depends on concomitant risk factors. Diabetes Care. 2006;29(2):391-7.

3. Bulugahapitiya $U$, et al. Is diabetes a coronary risk equivalent? Systematic review and meta-analysis. Diabet Med. 2009;26(2):142-8.

4. Stamler J, Wentworth D, Neaton JD. Is relationship between serum cholesterol and risk of premature death from coronary heart disease continuous and graded? Findings in 356,222 primary screenees of the Multiple Risk Factor Intervention Trial (MRFIT). JAMA. 1986;256(20):2823-8.

5. Kannel WB, et al. Serum cholesterol, lipoproteins, and the risk of coronary heart disease. The Framingham study. Ann Intern Med. 1971;74(1):1-12.

6. Feingold KR. Cholesterol lowering drugs, in Endotext. Feingold KR (Eds). MDText.com, Inc. Copyright (c) 2000-2020, MDText.com, Inc.: South Dartmouth (MA). 2000
7. Baigent C, et al. Efficacy and safety of more intensive lowering of LDL cholesterol: a meta-analysis of data from 170,000 participants in 26 randomised trials. Lancet. 2010;376(9753):1670-81.

8. Soran H, Schofield JD, Durrington PN. Cholesterol, not just cardiovascular risk, is important in deciding who should receive statin treatment. Eur Heart J. 2015;36(43):2975-83.

9. ESC/EAS guidelines for the management of dyslipidaemias. Lipid modification to reduce cardiovascular risk. Atherosclerosis. 2019;290:140-205.

10. Pyŏrälä K, et al. Cholesterol lowering with simvastatin improves prognosis of diabetic patients with coronary heart disease. A subgroup analysis of the Scandinavian Simvastatin Survival Study (4S). Diabetes Care. 1997;20(4):614-20.

11. Collins R, et al. MRC/BHF Heart Protection Study of cholesterol-lowering with simvastatin in 5963 people with diabetes: a randomised placebo-controlled trial. Lancet. 2003;361(9374):2005-16.

12. Long-Term Intervention with Pravastatin in Ischaemic Disease LIPID Study Group. Prevention of cardiovascular events and death with pravastatin in patients with coronary heart disease and a broad range of initial cholesterol levels. N Engl J Med. 1998;339(19):1349-57.

13. Disease C, Management R. Standards of medical care in diabetes-2021. Diabetes Care. 2021;44(Supplement 1):S125-50.

14. NICE, type 2 diabetes in adults: management [CG28]. 2015

15. NICE, type 1 diabetes in adults: diagnosis and management [NG17]. 2015

16. Soran $\mathrm{H}$, et al. Quantitative evaluation of statin effectiveness versus intolerance and strategies for management of intolerance. Atherosclerosis. 2020;306:33-40.

17. Collins $\mathrm{R}$, et al. Interpretation of the evidence for the efficacy and safety of statin therapy. Lancet. 2016;388(10059):2532-61.

18. Tobert JA, Newman CB. The nocebo effect in the context of statin intolerance. Amsterdam: Elsevier; 2016.

19. Stroes ES, et al. Statin-associated muscle symptoms: impact on statin therapy-European Atherosclerosis Society consensus panel statement on assessment, aetiology and management. Eur Heart J. 2015;36(17):1012-22. 
20. Mach F, et al. Adverse effects of statin therapy: perception vs the evidence-focus on glucose homeostasis, cognitive, renal and hepatic function, haemorrhagic stroke and cataract. Eur Heart J. 2018;39(27):2526-39.

21. Adhyaru BB, Jacobson TA. Safety and efficacy of statin therapy. Nate Rev Cardiol. 2018;1:1.

22. Tobert JA, Newman CB. The nocebo effect in the context of statin intolerance. J Clin Lipidol. 2016;10(4):739-47.

23. Mach F, et al. Adverse effects of statin therapy: perception vs the evidence-focus on glucose homeostasis, cognitive, renal and hepatic function, haemorrhagic stroke and cataract. Eur Heart J. 2018;39(27):2526-39.

24. Adhyaru BB, Jacobson TA. Safety and efficacy of statin therapy. Nat Rev Cardiol. 2018;15(12): 757-69.

25. Sampson UK, Linton MF, Fazio S. Are statins diabetogenic? Curr Opin Cardiol. 2011;26(4):342-7.

26. Sattar N, Taskinen MR. Statins are diabetogenic-myth or reality? Atheroscler Suppl. 2012;13(1):1-10.

27. Masson W, et al. Effect of bempedoic acid on new onset or worsening diabetes: a meta-analysis. Diabetes Res Clin Pract. 2020;168:108369.

28. Clinicaltrials.gov. 2020; Available from: https:// clinicaltrials.gov/ct $2 /$ results?cond=\&term $=$ bempedoic+acid+AND+bempedoic+acid\&cntry= \&state $=\&$ city $=\&$ dist $=$.

29. Ray KK, et al. Safety and efficacy of bempedoic acid to reduce LDL cholesterol. N Engl J Med. 2019;380(11):1022-32.

30. Goldberg AC, et al. Effect of bempedoic acid vs placebo added to maximally tolerated statins on low-density lipoprotein cholesterol in patients at high risk for cardiovascular disease: the CLEAR wisdom randomized clinical trial. JAMA. 2019;322(18):1780-8.

31. Ballantyne CM, et al. Efficacy and safety of bempedoic acid added to ezetimibe in statin-intolerant patients with hypercholesterolemia: a randomized, placebo-controlled study. Atherosclerosis. 2018;277:195-203.

32. Laufs U, et al. Efficacy and safety of bempedoic acid in patients with hypercholesterolemia and statin intolerance. J Am Heart Assoc. 2019;8(7):e011662.

33. Cicero AFG, et al. Efficacy and safety of bempedoic acid for the treatment of hypercholesterolemia: a systematic review and meta-analysis. PLoS Med. 2020;17(7):e1003121-e1003121.

34. Wang X, et al. Efficacy and safety of bempedoic acid for prevention of cardiovascular events and diabetes: a systematic review and meta-analysis. Cardiovasc Diabetol. 2020;19(1):128.

35. Markham A. Bempedoic acid: first approval. Drugs. 2020;80(7):747-53.

36. Pinkosky SL, et al. AMP-activated protein kinase and ATP-citrate lyase are two distinct molecular targets for ETC-1002, a novel small molecule regulator of lipid and carbohydrate metabolism. J Lipid Res. 2013;54(1):134-51.

37. Cramer CT, et al. Effects of a novel dual lipid synthesis inhibitor and its potential utility in treating dyslipidemia and metabolic syndrome. J Lipid Res. 2004;45(7):1289-301.

38. Ballantyne CM, et al. ETC-1002 lowers LDL-C and beneficially modulates other cardio-metabolic risk factors in hypercholesterolemic subjects with either normal or elevated triglycerides. J Am Coll Cardiol. 2012;59(13_Supplement):E1625-E1625.

39. Li Y, et al. Hs-CRP and all-cause, cardiovascular, and cancer mortality risk: a meta-analysis. Atherosclerosis. 2017;259:75-82.

40. Bohula EA, et al. Inflammatory and cholesterol risk in the FOURIER trial. Circulation. 2018;138(2): $131-40$.

41. Ridker PM, et al. Rosuvastatin to prevent vascular events in men and women with elevated C-reactive protein. N Engl J Med. 2008;359(21):2195-207.

42. Yetley EA, Harlan WR Jr. Surrogate disease markers as substitutes for chronic disease outcomes in studies of diet and chronic disease relations. Am J Clin Nutr. 2017;106(5):1175-89.

43. Naeem FMG, Fisher M. Cardiovascular outcomes trials with non-statin lipid-lowering drugs in diabetes. Br J Diabetes. 2018;18:101-5.

44. Soran $\mathrm{H}$, et al. Evidence for more intensive cholesterol lowering. Curr Opin Lipidol. 2017;28(4): 291-9.

45. Ballantyne CM, et al. Bempedoic acid plus ezetimibe fixed-dose combination in patients with hypercholesterolemia and high CVD risk treated with maximally tolerated statin therapy. Eur J Prev Cardiol. 2020;27(6):593-603.

46. Navar AM, et al. Prevalence and management of symptoms associated with statin therapy in community practice: insights from the PALM (patient 
and provider assessment of lipid management) registry. Circ Cardiovasc Qual Outcomes. 2018;11(3):e004249.

47. Engeda JC, et al. Evidence of heterogeneity in statin-associated type 2 diabetes mellitus risk: a metaanalysis of randomized controlled trials and observational studies. Diabetes Res Clin Pract. 2019;151: 96-105.

48. Mao J, Blumenthal RS, Martin SS. Bempedoic Acid, the Next LDL Cholesterol-Lowering Medication to Join the Arsenal? Insights From the CLEAR Wisdom Trial. 2019.

49. EMC. Nilemdo 180mg film-coated tablets. 2020; Available from: https://www.medicines.org.uk/ emc/product/11743/smpc\#gref.

50. Kaltwasser JLU. Bempedoic acid lowers LDL cholesterol in statin-intolerant patients. 2019

51. Khunti KDM, Kutikova L, Catterick D, Sorio-Vilela $\mathrm{F}$, Gleeson $\mathrm{M}$, et al. Association of a combined measure of adherence and treatment intensity with cardiovascular outcomes in patients with atherosclerosis or other cardiovascular risk factors treated with statins and/or ezetimibe. JAMA Netw Open. 2018;1(8):e185554.

52. do Rezende Macedo Nascimento RCMT, Godman B, MacBride Stewart S, Hurding S, de Acurcio Assis F, et al. Real-world evaluation of the impact of statin intensity on adherence and persistence to therapy: a Scottish population-based study. Br J Clin Pharmacol. 2020;86(12):2349-61.

53. NICE. Evolocumab for treating primary hypercholesterolaemia and mixed dyslipidaemia. 2016; Available from: https://www.nice.org.uk/guidance/ ta394.

54. Morieri ML, Avogaro A, Fadini GP, et al. Cholesterol lowering therapies and achievement of targets for primary and secondary cardiovascular prevention in type 2 diabetes: unmet needs in a large population of outpatients at specialist clinics. Cardiovasc Diabetol. 2020;19:190. https://doi.org/10.1186/ s12933-020-01164-8. 